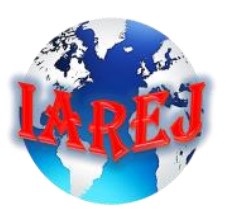

Research Article

\title{
Nonlinear vibration and dynamic response of nano composite conical tube by conveying fluid flow
}

\author{
Masoud Rahmani ${ }^{a * *}$ (D) and Amin Moslemi Petrudi ${ }^{a}$ (iD) \\ ${ }^{a}$ Department of Mechanical Engineering, Tehran University, Tehran, Iran
}

\begin{tabular}{l}
\hline ARTICLE INFO \\
\hline Article history: \\
Received 09 June 2020 \\
Revised 18 July 2020 \\
Accepted 29 July 2020 \\
\hline Keywords: \\
Conical tube \\
Fluid flow \\
Galerkin method \\
Vibrations
\end{tabular}
\begin{abstract}
Modeling of tubes containing fluid flow is widely used in the study of heat exchangers, nuclear reactors, micro and nano tools, etc. This system is structurally simple but very complex in terms of dynamic behavior and vibrations. In this paper, an analytical relationship for nonlinear vibrations of self-excitation of a nanocomposite conical tube containing fluid flow is extracted, one end of which is free and the other side is fixed and is under gravitational force. The base material is assumed to be 1200 series aluminum, which is reinforced with carbon nanotubes. The Hamiltonian equations are obtained, assuming the Euler-Bernoulli beam theory and the use of the Galerkin method, dissected the partial derivative equations into Ordinary Differential Equations (ODE), then solved by MATLAB coding and investigated the effect of various parameters on system behavior. As the fluid velocity increases, the amplitude of the vibration increases and the nonlinear effects of the system increase, so more modes are needed to converge the responses. In a conical tube, the $\beta_{\mathrm{T}}$ coefficient increases with increasing inner diameter along the tube and the system becomes more stable. Increasing the length of the pipe makes the opening conical pipe more stable and the closing conical pipe more unstable. The change in length has no effect on the stability of the cylindrical tube.
\end{abstract}

(C) 2020, Advanced Researches and Engineering Journal (IAREJ) and the Author(s).

\section{Introduction}

The issue of fluid flow pipes has been seriously studied since about 1950 to analyze the vibrations of oil pipelines. Although this system is structurally simple, it is very complex in terms of dynamic behavior. Various nonlinear phenomena are more pronounced in the dynamic behavior of these systems, especially in tubes with free ends. Modeling of such systems is used in heat exchangers, nuclear reactors, micro and nano tools, robots and underwater equipment, etc. One of the main features of these systems is that if the velocity of the material being transferred is high enough, the structure may suffer from deflection or oscillation instability in bending modes. For these structures, the rate of acceleration that leads to the onset of instability is called the critical mass transfer rate. In analyzing such systems, it is usually to determine two cases. The first is the critical velocity of the mass being transferred and the second is the relation between the characteristic frequencies (specific values) and the velocity of the transfer mass. The first is the main goal of sustainability analysis, and the second is important if the characteristics of the free frequency and the response to a set of specific excitation are considered. Extensive research has been done on various types of tubes containing fluid flow, but most of this research has been limited to linear models and a small number of variable cross-sections. Probing biomechanical properties with a centrifugal force quartz crystal microbalance [1]. This study examines flexible tubes containing flow. Lu et al. [2] investigated internal resonance and stress distribution of pipes conveying fluid in supercritical regime. Xinbo [3] showed in experimental device for the study of liquidsolid coupled flutter instability of salt cavern leaching tubing. Amabili et al. [4] studied the effects of geometric defects on the nonlinear stability of a cylindrical shell containing fluid flow and the effect of Van der Waals force. Liang et al. [5] analyzing the vibrations caused by

* Corresponding author. Tel.: +9801142565736; Fax: +9801142565736

E-mail addresses: msrahmani@ihu.ac.ir (M.Rahmani),amin.tehran2020@gmail.com (A. Moslemi Petrudi)

ORCID: 0000-0002-0519-0670 (M. Rahmani), 0000-0002-5928-0479 (A. Moslemi Petrudi)

DOI: $10.35860 /$ iarej. 750166 
the flow and stability of double-walled carbon nanotubes based on the theory of coupling stress, investigated the side and fixed effect of Winkler elastic constant (spring stiffness). Sadeghi et al. [6] investigated the effect of mass at the end of the tube for three-dimensional mode. Ghayesh et al. [7] studied the motion of a tube integrated containing the flow, taking into account the flexibility of the tube in two dimensions. One of the areas that has received the most attention recently is the nanoscale and the use of nanocomposites. Farajpour et al. [8] investigated the nonlinear mechanics of nanotubes containing fluid flow. Liang et al. [9] investigated the free vibrations and dynamics of the rotating tube containing the fluid flow. Their study found that rotational motion reinforces tube strength and eliminates the buckling instability. Lu et al. [10] investigated the effects of nonlinear vibrations on the fatigue life of fluid transfer tubes made of Functionally Graded Material (FGM) materials. The results of fatigue analysis show that internal resonance shortens the life of fatigue and reduces the distribution coefficient of FGM tube, reduces resonance response and maximum tube tension. Mohammadi et al. [11] investigated an efficient solver for fully coupled solution of interaction between incompressible fluid flow and nanocomposite truncated conical shells. the dynamic instabilities of nanocomposite truncated conical shells containing a quiescent or a flowing inviscid fluid are scrutinized. Nonlinear dynamic equations are established according to the Novozhilov's nonlinear shell theory along with Green's strains and Hamilton principle. Dinh et al. [12] investigated for electro-thermo-mechanical vibration of nanocomposite cylindrical shells with an internal fluid flow. Khudayarov et al. [13] investigated numerical simulation of vibration of composite pipelines conveying fluids with account for lumped masses. Sedighi [14] investigated divergence and flutter instability of magneto-thermo-elastic Cubic boron nitride (C-BN) hetero-nanotubes conveying fluid. On the basis of finite element analysis, an eigenvalue problem is performed to examine the vibrational characteristics of a hetero-nanotube made of $\mathrm{C}$-BN nanotubes in magnetic and thermal environment. Bahaadini et al. [15] investigated dynamic stability of viscoelastic nanotubes conveying pulsating magnetic Nano flow under magnetic field. Dynamic stability analysis of viscoelastic carbon nanotubes (CNTs) conveying pulsating magnetic Nano flow subjected to a longitudinal magnetic field. Li et al. [16] analytically investigated Vibration of a FluidConveying Pipe Flexibly Restrained at the Ends. In their studies the effects of different spring stiffness coefficients on the parametric resonance responses are presented. According to a review of similar research, the nonlinear vibrations of the tube with the variable cross-section have not been analyzed analytically. In this study, a nonlinear analytical relation is obtained for the self-excitation vibrations of the nanocomposite conical tube with a free end that contains a fluid flow and is under gravity.

\section{Problem Statement}

Figure 1 shows the geometric parameters of the tube. In this study, it is assumed that the fluid is incompressible, so the volumetric flow rate is constant along the tube and the velocity obtained by dividing the flow rate by the fluid cross section. The total cross-sectional area at each point is determined by Ae (s), the area of the section containing the Af (s) fluid, and the cross-sectional area of the tube with Ap (s). The tube is assumed to be a fixed head. The flow entry's the tube from the top and is under gravity and exits from the bottom of the tube.

The equations of motion in the form of a partial differential equation derivative are a function of $\mathrm{x}$ and $\mathrm{t}$, which must be written to the matrix form of the finite element to solve the equations. The matrix form can be solved in a variety of ways, including the Runge-Kutta method. The steps for converting equations to the matrix form of finite element components are as follows, that is, first, using the Galerkin method, write the equations of the Partial Differential Equations as a weak form, and then the common matrix that you see these steps in Figure 2.

The Galerkin method is a special case of the residual weight method, which is a function of weight equal to the approximation function.

\subsection{Fourth Order Runge-Kutta Method}

This method has several levels that in this article, the fourth order mode is used. Fourth Order Runge-Kutta Method is a numerical technique used to solve ordinary differential equation of the form.

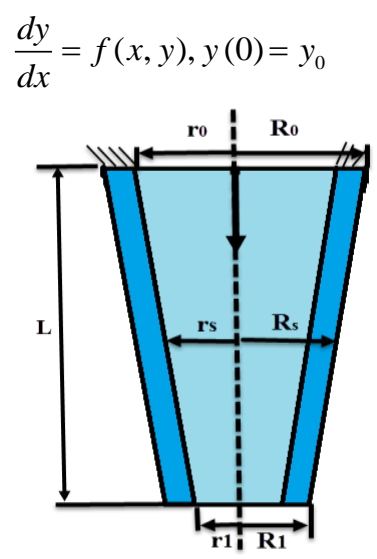

Figure 1. Geometry of the problem

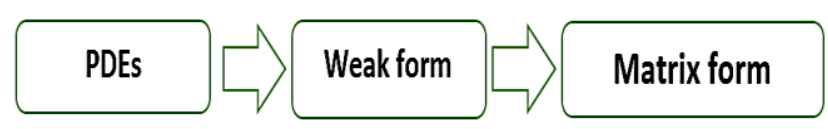

Figure 2. Steps to convert partial derivative equations into a matrix form 
$\mathrm{K}_{1}, \mathrm{~K}_{2}, \mathrm{~K}_{3}$, and $\mathrm{K}_{4}$ are the values of the Runge-Kutta method, which should be weighted average of these values with the values of displacement and velocity in the previous step in order to calculate the velocity and displacement in each step [17].

$$
\begin{aligned}
& k_{1}=h f\left(x_{i}, y_{i}\right), k_{2}=h f\left(x_{i}+\frac{h}{2}, y_{i}+\frac{k_{1}}{2}\right), \\
& k_{3}=h f\left(x_{i}+\frac{h}{2}, y_{i}+\frac{k_{2}}{2}\right), \\
& k_{4}=h f\left(x_{i}+h, y_{i}+k_{3}\right) y_{i+1} \\
& =y_{i}+\frac{1}{6}\left(k_{1}+2 k_{2}+2 k_{3}+k_{4}\right)
\end{aligned}
$$

in which $\mathrm{h}$ is time step of solving. Classical elasticity theories deal with issues in which the displacements are small, but in the case under consideration it is possible to make large displacements, which makes the importance of nonlinear terms important. In elasticity $(\mathrm{x}, \mathrm{y}, \mathrm{z})$ it indicates the position of the material point in the elastic body and ( $u$, $\mathrm{v}, \mathrm{w})$ corresponds to the displacements of the same material point. Properties of composite material reinforced by carbon nanotube are obtained using the following relations [18]:

$$
\begin{aligned}
& E_{11_{C}}=\eta_{1} V_{C N T} E_{11}{ }^{C N T}+V_{m} E^{m} \\
& \frac{\eta_{2}}{E_{22_{C}}}=\frac{V_{C N T}}{E_{22}{ }^{C N T}}+\frac{V_{m}}{E_{m}} \\
& \frac{\eta_{3}}{G_{12_{C}}}=\frac{V_{C N T}}{G_{12}{ }^{C N T}}+\frac{V_{m}}{G^{m}} \\
& v_{12_{C}}=V_{C N T} v_{12}{ }^{C N T}+V_{m} v^{m} \\
& \rho_{0_{C}}=V_{C N T} \rho_{0}{ }^{C N T}+V_{m} \rho_{0}{ }^{m} \\
& a_{11_{C}}=V_{C N T} a_{11}{ }^{C N T}+V_{m} a^{m} \\
& a_{22_{C}}=\left(1+v_{12}^{C N T}\right) V_{C N T} a_{22}{ }^{C N T}+\left(1+v^{m}\right) V_{m} a^{m}-v_{12} a_{11 c}
\end{aligned}
$$

In this relation $E_{11}^{C N T}$ and $E_{22}^{C N T}$ are elastic modulus and $G_{12}^{C N T}$ shear modulus of single-walled carbon nanotubes. $\eta_{1}, \eta_{2}$ and $\eta_{3}$ are the carbon nanotube performance parameters. $V_{C N T}$ and $V_{m}$, respectively, are the volume fraction of carbon nanotubes and matrices that apply to the $V_{C N T}+V_{m}=1$ relation. $v_{12}^{C N T}$ and $\rho_{0}^{C N T}$ are Poisson's ratio and density of carbon nanotubes $\nu^{m}$ and $\rho_{0}^{m}$ are Poisson's ratio and density of matrix. In these relations, $\alpha_{11}^{C N T} . \alpha_{22}^{C N T}$ and $\alpha^{m}$ are the thermal expansion coefficients of the carbon nanotube and the matrix. The properties of carbon nanotubes are presented in Table 1 and the values of carbon nanotube performance parameters are presented in Table 2 .

The base material is assumed to be 1200 series aluminum, which is reinforced with carbon nanotubes. The fluid passing through the pipe is also assumed to be water. The main mechanical properties of aluminum are listed in Table 3.

The assumption of the problem is that the fluid is incompressible and turbulent that the diameter of the tube is small compared to the length. Assuming the EulerBernoulli beam theory was used for the problem, rotational inertia and shear deformation were ignored, assuming that the central axis of the tube did not change in length (the condition of non-expansion). The energy method is based on Hamilton's principle and is as follows:

$$
\delta \int_{t 1}^{t 2} L d t+\int_{t 1}^{t 2} \delta W d t=0
$$

In this relation, $\mathrm{L}$ is the Lagrangian system $\left(\mathrm{L}=\mathrm{T}_{\mathrm{P}}+\mathrm{T}_{\mathrm{F}}-\right.$ $V_{P}-V_{F}$ ) where $T_{P}$ and $V p$ are kinetic energy and tube potential and $T_{F}$ and $V_{F}$ are kinetic energy and fluid potential, respectively. $\delta W$ virtual work is a force that is not included in Lagrangian. But Hamilton's original form is established for closed systems, that is, in a system where there is no mass flow in or out, the Hamilton principle should be used to investigate the problem, that the system is assumed to be independent of the lost forces $\delta W=0$ :

$$
\delta \int_{t 1}^{t 2} L d t=\int_{t 1}^{t 2}\left[M U\left(\frac{\partial \vec{R}}{\partial t}+U \vec{\tau}\right) \cdot \partial \vec{R}\right] d t
$$

$\vec{T}$ and $\vec{R}$ are the position vectors and the vector unit tangent to the tube, respectively. The relation was established in 1961 by Benjamin [21] about flow tube that was modeled with the Euler-Bernoulli beam. The MU term coefficient is the amount of energy that is added to or reduced from the system by the end of the tube, the right side of the relation was considered as the transfer virtual momentum through the free surface of the end of the tube. This term is directly related to the instability mechanism. The energy finding by the tube is as follows:

$$
\Delta W=-\int_{0}^{t 1} M U\left(\dot{R}^{2}+U \vec{\tau} \cdot \vec{R}\right) d t
$$

Table 1. Material properties of single-walled carbon nanotube $(10,10)\left(v_{12}^{C N T}=0.175\right)[19]$

\begin{tabular}{|c|c|c|c|}
\hline Temperature $(\mathrm{K})$ & 700 & 500 & 300 \\
\hline$E_{11}^{C N T}(\mathrm{TPa})$ & 5.4744 & 5.5308 & 5.6466 \\
\hline$E_{22}^{C N T}(\mathrm{TPa})$ & 6.8641 & 6.9348 & 7.0800 \\
\hline$G_{12}^{C N T}(\mathrm{TPa})$ & 1.9644 & 1.9643 & 1.9445 \\
\hline$\alpha_{11}^{C N T}\left(10^{-} 6 / \mathrm{K}\right)$ & 4.6677 & 4.5361 & 3.4584 \\
\hline$\alpha_{2}^{C N T}\left(10^{-6} / \mathrm{K}\right)$ & 4.8943 & 8.0189 & 5.1682 \\
\hline
\end{tabular}

Table 2. Efficiency parameters for different values of VCNT [19]

\begin{tabular}{|c|c|c|c|}
\hline$\eta_{3}$ & $\eta_{2}$ & $\eta_{1}$ & $V_{\text {CNT }}$ \\
\hline 0.934 & 0.934 & 0.149 & 0.11 \\
\hline 0.941 & 0.941 & 0.150 & 0.14 \\
\hline 1.381 & 1.381 & 0.149 & 0.17 \\
\hline
\end{tabular}


Table 3. Mechanical properties of 1200 series aluminum [20]

\begin{tabular}{|c|c|c|c|c|}
\hline properties & $\mathrm{E}(\mathrm{Gpa})$ & $\mathrm{G}(\mathrm{Gpa})$ & $\rho\left(\mathrm{g} / \mathrm{cm}^{3}\right)$ & $v$ \\
\hline value & 70 & 28 & 2.7 & 0.29 \\
\hline
\end{tabular}

If the two ends of the tube are fixed $\Delta W=0$ and the system is conservative. For small input velocity's, the fluid is $\Delta W<0$ and the system is stable, but for large enough velocity's, the value of $\Delta W$ is positive and the system is unstable. Defining an exact relation for kinetic energy is very important in large deformations. Stoker [22] introduces the following relation for this purpose:

$$
V=\frac{E}{2} \int_{0}^{L}\left[A \varepsilon^{2}+1(1+\varepsilon)^{2} k^{2}\right] d x
$$

$X$ the Lagrangian coordinates, $A$ cross-sectional area, $I$ moment of inertia, and $\varepsilon$ strain. The tube with the assumption of non-expandability $\varepsilon=0$, the relation is as follows:

$$
V=\frac{E I}{2} \int_{0}^{L} k^{2} d x=\frac{E I}{2} \int_{0}^{L} k^{2} d s
$$

Gravitational energy is dependent on mass and is in a uniform field as follows:

$$
G=\int \rho g x d V
$$

The gravitational energy in the present case is written as follows:

$$
G=-(m+M) g \int_{0}^{L}(X+u) d s
$$

The kinetic energy of the whole system is the sum of the kinetic energy of the tube, $T_{p}$, and the kinetic energy of the $\mathrm{T}_{\mathrm{F}}$ fluid, which is defined as follows:

$$
T_{P}=\frac{m}{2} \int_{0}^{L} V_{P}^{2} d s \quad, \quad T_{F}=\frac{m}{2} \int_{0}^{L} V_{F}^{2} d s
$$

Using the Hamilton principle, after performing the necessary calculations, the equation of motion is obtained as follows (for a fixed cross-sectional area):

$$
\begin{aligned}
& (m+M) \ddot{y}+2 M U \dot{y}^{\prime}\left(1+y^{2}\right)+ \\
& (m+M) g y^{\prime}\left(1+\frac{1}{2} y^{2}\right)+y^{\prime \prime}\left(M U^{2}\left(1+y^{2}\right)\right. \\
& +\left(M \dot{U}-(m+M) g(L-s)\left(1+\frac{3}{2} y^{2}\right)\right) \\
& +E I\left(y^{\prime \prime \prime}\left(1+y^{2}\right)+4 y^{\prime} y^{\prime \prime} y^{\prime \prime \prime}+y^{\prime \prime 3}\right) \\
& -y^{\prime \prime}\left(\int_{S}^{L} \int_{0}^{S}(m+M)\left(\dot{y}^{2}+y^{\prime} \ddot{y}^{\prime}\right) d s d s\right. \\
& +\int_{s}^{L}\left(\frac{M U^{2}}{2} y^{2}+2 M U y^{\prime} \dot{y}^{\prime}\right. \\
& \left.\left.+M U^{2} y^{\prime} y^{\prime \prime}\right) d s\right)+y^{\prime} \int_{0}^{S}(m+M)\left(\dot{y}^{2}+y^{\prime} \ddot{y}^{\prime}\right) d s=0
\end{aligned}
$$

Assuming the steady flow is the dimensionless linear equation as follows:
Table 4. Physical concepts in the equation of motion.

\begin{tabular}{|c|c|}
\hline Phrase & Physical concept \\
\hline$(m+M) \frac{\partial^{2} y}{\partial t^{2}}$ & Inertial force \\
\hline $2 M U \frac{\partial^{2} y}{\partial x \partial t}$ & Coriolis force \\
\hline$M U^{2} \frac{\partial^{2} y}{\partial x^{2}}$ & Center side force \\
\hline$-(m+M) g\left((L-x) y^{\prime}\right)^{\prime}$ & The force of gravity \\
\hline$E I \frac{\partial^{4} y}{\partial x^{4}}$ & Durable bending force \\
\hline$M \frac{d U}{d t}$ & The force of the unstable flow \\
\hline
\end{tabular}

$$
\begin{gathered}
\eta^{\prime \prime \prime}+\ddot{\eta}+2 u \sqrt{\beta} \dot{\eta}^{\prime}+\eta^{\prime \prime}\left(u^{2}-\gamma(1-\xi)\right)+\gamma \eta^{\prime} \\
=0
\end{gathered}
$$

Which are its dimensionless parameters:

$$
\begin{aligned}
\xi & =\frac{x}{L} \\
\eta & =\frac{y}{L} \\
\tau & =\left(\frac{E I}{m+M}\right)^{1 / 2} \frac{t}{L^{2}} \\
\gamma & =\frac{m+M}{E I} L^{3} g \\
\beta & =\frac{M}{m+M}
\end{aligned}
$$

Using these dimensionless parameters, Equation 18 becomes the following form:

$$
\begin{aligned}
& \eta^{\prime \prime \prime}+\ddot{\eta}+2 u \sqrt{\beta} \dot{\eta}^{\prime}\left(1+\eta^{2}\right)+\eta^{\prime \prime}\left(u^{2}\left(1+\eta^{2}\right)\right. \\
& \left.+(\dot{u} \sqrt{\beta}-\gamma)(1-\xi)\left(1+\frac{3}{2} \eta^{2}\right)\right)+\gamma \dot{\eta}\left(1+\frac{1}{2} \eta^{2}\right) \\
& +\eta^{\prime \prime \prime \prime} \eta^{2}+4 \eta^{\prime} \eta^{\prime \prime} \eta^{\prime \prime \prime}+\eta^{3}-\eta^{\prime \prime}\left(\int_{\xi}^{1} \int_{0}^{\xi}\left(\dot{\eta}^{2}+\eta^{\prime} \ddot{\eta}^{\prime}\right) d \xi d \xi\right. \\
& \left.+\int_{\xi}^{1}\left(\frac{\dot{u} \sqrt{\beta}}{2} \eta^{2}+2 u \sqrt{\beta} \eta^{\prime} \dot{\eta}^{\prime}+u^{2} \eta^{\prime} \eta^{\prime \prime}\right) d \xi\right) \\
& +\eta^{\prime} \int_{0}^{\xi}\left(\dot{\eta}^{2}+\eta \eta\right) d \xi d \xi=0
\end{aligned}
$$

Physically, $u$ is the dimensionless velocity of fluid, $\gamma$ is the ratio of gravitational force to bending strength, and $\beta$ is the ratio of fluid mass to total mass per unit length of pipe. Positive values of $\gamma$ mean that the tube is hanging, ie the upper end of the tube is fixed and the lower end of the tube is free. Negative $\gamma$ values mean that the lower end of the tube is fixed and the upper end of the tube is free. Considering the change in cross section, the equation of motion is as follows: 


$$
\begin{aligned}
& \left(m_{s}+M_{s}\right) \ddot{y}+2 M_{s} U_{s} \dot{y}^{\prime}\left(1+y^{2}\right)+ \\
& \left(m_{s}+M_{s}\right) g y^{\prime}\left(1+\frac{1}{2} y^{2}\right)+y^{\prime \prime}\left(M_{L} U_{L}^{2}\left(1+y^{2}\right)\right. \\
& -g\left(1+\frac{3}{2} y^{2}\right) \int_{s}^{L}\left(m_{s}+M_{s}\right) d s \\
& +E I_{s}\left(y^{\prime \prime \prime \prime}+4 y^{\prime} y^{\prime \prime} y^{\prime \prime \prime}+y^{3}+y^{\prime \prime \prime} y^{2}\right) \\
& +E I_{s^{\prime}}\left(2 y^{\prime \prime \prime}+2 y^{\prime \prime \prime} y^{2}+3 y^{2} y^{\prime}\right)+E I_{S}^{\prime \prime}\left(y^{\prime \prime}+y^{\prime \prime} y^{2}\right) \\
& -y^{\prime \prime}\left(\int_{S}^{L}\left(m_{s}+M_{s}\right) \int_{0}^{S}\left(\dot{y}^{2}+y^{\prime} \ddot{y}^{\prime}\right) d s d s\right. \\
& \left.+\int_{S}^{L}\left(2 M_{s} U_{s} y^{\prime} \dot{y}^{\prime}+M_{L} U_{L}^{2} y^{\prime} y^{\prime \prime}\right) d s\right) \\
& \left.+y^{\prime}(m+M) \int^{S} \dot{y}^{2}+y^{\prime} \ddot{y}^{\prime}\right) d s=0
\end{aligned}
$$

The geometric characteristics of the tube is shown in Figure 1. Geometric dimensionless parameters are written based on the geometric characteristics of the tube. The moment of inertia and mass, the cross-sectional area and density are dimensionless and the relations related to the radius and dimensionless dimension are obtained as:

$$
\begin{aligned}
& r_{s}=r .+\frac{r \cdot-r_{1}}{L} s, \\
& R_{s}=R .+\frac{R \cdot-R_{1}}{L} s \\
& A_{e}(s)=\pi R_{s}^{2}, A_{f}(s)=\pi r_{s}^{2}, \\
& A_{p}(s)=A_{e}(s)-A_{f}(s)=\pi\left(R_{s}{ }^{2}-r_{s}^{2}\right)
\end{aligned}
$$

By dividing the area and moment of inertia by their values at the beginning of their tube, it becomes dimensionless:

$$
\begin{aligned}
& A_{e}{ }^{*}=\frac{A_{e}(s)}{A_{e}(0)}=\frac{R_{s}{ }^{2}}{R_{0}{ }^{2}}, A_{f}{ }^{*}=\frac{A_{f}(s)}{A_{f}(0)}=\frac{r_{s}{ }^{2}}{R_{0}{ }^{2}}, \\
& A_{p}{ }^{*}=\frac{A_{p}(s)}{A_{e}(0)}=\frac{R_{s}{ }^{2}-r_{s}{ }^{2}}{R_{0}{ }^{2}} \\
& I^{*}=\frac{I(s)}{I(0)}=\frac{R_{s}{ }^{4}-r_{s}{ }^{4}}{R_{0}{ }^{4}-r .^{4}} \\
& x=\frac{L}{R .}, \lambda=\frac{r .}{R .}, a_{i}=\frac{r \cdot-r_{1}}{L}, \\
& a_{e}=\frac{R \cdot-R_{1}}{L}, \xi=\frac{s}{L}, \eta=\frac{y}{L}
\end{aligned}
$$

Therefore, the area and time of the dimensionless moment of inertia are as follows:

$$
\begin{aligned}
& A_{f}^{*}(\xi)=a_{i}^{2} x^{2} \xi^{2}-2 a_{i} \lambda x \xi+\lambda^{2} \\
& A_{e}^{*}(\xi)=a_{e}^{2} x^{2} \xi^{2}-2 a_{e} \lambda x \xi+1 \\
& A_{p}^{*}(\xi)=x^{2}\left(a_{e}^{2}-a_{i}^{2}\right) \xi^{2} \\
& +2 x\left(a_{i} \lambda-a_{e}\right) \xi+1-\lambda^{2} \\
& I^{*}(\xi)=\frac{x^{4}\left(a_{e}^{4}-a_{i}^{4}\right)}{1-\lambda^{4}} \lambda^{4}+\frac{4 x^{3}\left(a_{i}^{3}-a_{e}^{3}\right)}{1-\lambda^{4}} \xi^{3} \\
& +\frac{6 x^{2}\left(a_{e}^{2}-a_{i}^{2} \lambda^{2}\right)}{1-\lambda^{4}} \xi^{2}+\frac{4 x\left(a_{i} \lambda^{3}-a_{e}\right)}{1-\lambda^{4}} \xi+1
\end{aligned}
$$

Other dimensionless parameters are defined as follows:

$$
\begin{aligned}
& p^{*}=\frac{\rho_{p}}{p_{f}}, u=\left(\frac{p_{f} A_{f}}{E I}\right)_{\xi=0}^{\frac{1}{2}} U . L, \\
& \tau=\left(\frac{E I}{\rho_{p} A_{p}+\rho_{f} A_{f}}\right)_{\xi=0}^{\frac{1}{2}} \frac{t}{L^{2}}, \\
& \gamma=\left(\frac{\rho_{p} A_{p}+\rho_{f} A_{f}}{E I}\right)_{\xi=0}^{\frac{1}{2}} L^{3} g
\end{aligned}
$$

To shorten the equations, the following parameter is defined:

$$
\Lambda=\frac{\lambda^{2}}{\lambda^{2}\left(1-\rho^{*}\right)+\rho^{*}}
$$

Finally, dimensional equation in the form below:

$$
\begin{aligned}
& \frac{\Lambda}{\lambda^{2}}=\left(A_{f}^{*}+\rho^{*} A_{p}^{*}\right) \ddot{\eta}+2 u \sqrt{\Lambda} \dot{\eta}^{\prime}\left(1+\eta^{2}\right)+ \\
& \eta^{\prime \prime}\left(\frac{u^{2} \lambda^{2}}{A_{f}^{*}(1)}\left(1+\eta^{2}\right)-\right. \\
& \left.\left.\frac{\Lambda}{\lambda^{2}} \gamma\left(1+\frac{3}{2} \eta^{2}\right) \int_{\zeta}^{1} A_{f}^{*}+\rho^{*} A_{p}^{*}\right) d \xi\right) \\
& +\frac{\Lambda}{\lambda^{2}} \gamma\left(A_{f}^{*}+\rho^{*} A_{p}^{*}\right) \eta^{\prime}\left(1+\frac{1}{2} \eta^{2}\right)+ \\
& I^{*}\left(\eta^{\prime \prime \prime} \eta^{2}+4 \eta^{\prime} \eta^{\prime \prime} \eta^{\prime \prime \prime}+\eta^{3}+\eta^{\prime \prime \prime}\right)+ \\
& I^{* \prime}\left(2 \eta^{\prime \prime \prime}\left(1+\eta^{2}\right)+3 \eta^{2} \eta^{\prime}\right)+I^{* \prime \prime} \eta^{\prime \prime}\left(1+\eta^{2}\right) \\
& -\eta^{\prime \prime}\left(\frac{\Lambda}{\lambda^{2}} \int_{\xi}^{1}\left(A_{f}^{*}+\rho^{*} A_{p}^{*}\right) \int_{0}^{\zeta}\left(\dot{\eta}^{2}+\eta^{\prime} \ddot{\eta}^{\prime}\right) d \xi d \xi\right. \\
& \left.+\int_{\xi}^{1}\left(2 u \sqrt{\Lambda} \eta^{\prime} \dot{\eta}^{\prime}+\frac{u^{2} \lambda^{2}}{A_{f}^{*}(1)} \eta^{\prime} \eta^{\prime \prime}\right) d \xi\right)+ \\
& \frac{\Lambda}{\lambda^{2}}\left(A_{f}^{*}+\rho^{*} A_{p}^{*}\right) \eta^{\prime} \int_{0}^{\xi}\left(\dot{\eta}^{2}+\eta^{\prime} \ddot{\eta}^{\prime}\right) d \xi d \xi=0
\end{aligned}
$$

After obtaining the equations of motion, it is necessary to use the Galerkin method mentioned earlier to make the equations discrete and matrix. 


$$
\begin{aligned}
& m_{i j} \ddot{q}_{j}+c_{i j} \dot{q} j+k_{i j} q_{j}+B_{i j k l} q_{j} q_{k} q_{l}+ \\
& D_{i j k l} q_{j} q_{k} \dot{q}_{l}+E_{i j k l} q_{j} \dot{q}_{k} \dot{q}_{l}+F_{i j k l} q_{j} q_{k} \ddot{q}_{l}=0 \text {, } \\
& m_{i j}=\frac{\Lambda}{\lambda^{2}} \int_{0}^{1}\left(A_{f}^{*}+\rho^{*} A_{p}^{*}\right) \varphi_{i} \varphi_{i} d \xi \\
& c_{i j}=2 u \sqrt{\Lambda} \int_{0}^{1} \varphi_{i} \varphi_{i}^{\prime} d \xi \text {, } \\
& k_{i j}=\int_{0}^{1} I^{*} \varphi_{i} \varphi_{i}^{\prime \prime \prime} d \xi+2 \int_{0}^{1} I^{* \prime} \varphi_{i} \varphi_{i}^{\prime \prime \prime} d \xi+\int_{0}^{1} I^{* \prime \prime} \varphi_{i} \varphi_{i}^{\prime \prime} d \xi+ \\
& \frac{u^{2} \lambda^{2}}{A_{f}^{*}(1)} \int_{0}^{1} \varphi_{i} \varphi_{i}^{\prime \prime} d \xi+\frac{\Lambda}{\lambda^{2}} \gamma\left(\int_{0}^{1} A_{f}^{*}+\rho^{*} A_{p}^{*}\right) \varphi_{i} \varphi_{i}^{\prime} d \xi \\
& \left.\left.-\int_{0}^{1} \varphi_{i} \varphi_{i}^{\prime \prime} \int_{\xi}^{1} A_{f}^{*}+\rho^{*} A_{p}^{*}\right) d \xi\right) \\
& B_{i j k l}=\frac{u^{2} \lambda^{2}}{A_{f}^{*}(1)} \int_{0}^{1} \varphi_{i}\left(\varphi_{i}^{\prime} \varphi_{k}^{\prime} \varphi_{l}^{\prime \prime} \int_{\xi}^{1} \varphi_{k}^{\prime} \varphi_{l}^{\prime \prime}\right) d \xi \\
& +\frac{\Lambda}{\lambda^{2}} \gamma\left(\frac{1}{2} \int_{0}^{1} A_{f}^{*}+\rho^{*} A_{p}^{*}\right) \varphi_{i}^{\prime} \varphi_{j}^{\prime} \varphi_{k}^{\prime} \varphi^{\prime}, d \xi \\
& -\frac{3}{2} \int_{0}^{1} \varphi_{i} \varphi_{j}^{\prime} \varphi_{k}^{\prime} \varphi_{l}^{\prime \prime} \int_{\xi}^{1}\left(A_{f}^{*}+\rho^{*} A_{p}^{*}\right) d \xi \\
& +\int_{0}^{1} I^{* \prime} \varphi_{i}\left(2 \varphi_{j}^{\prime} \varphi_{k}^{\prime} \varphi_{l}^{\prime \prime}+3 \varphi_{i} \varphi_{j}^{\prime} \varphi_{k}^{\prime \prime} \varphi_{l}^{\prime \prime}\right) d \xi \\
& +\int_{0}^{1} I^{* \prime \prime} \varphi_{i} \varphi_{j}^{\prime} \varphi_{k}^{\prime} \varphi_{l}^{\prime \prime} d \xi \\
& D_{i j k l}=2 u \sqrt{\Lambda} \int_{0}^{1} \varphi_{i}\left(\varphi_{j}^{\prime} \varphi_{k}^{\prime} \varphi_{l}^{\prime}-\right. \\
& \left.\varphi_{l}^{\prime \prime} \int_{\xi}^{1} \varphi_{k}^{\prime} \varphi_{l}^{\prime}\right) d \xi, E_{i j k l}=F_{i j k l}= \\
& \frac{\Lambda}{\lambda^{2}} \gamma\left(\int_{0}^{1}\left(A_{f}^{*}+\rho^{*} A_{p}^{*}\right) \varphi_{i} \varphi_{j}^{\prime} \int_{0}^{\xi} \varphi_{k}^{\prime} \varphi_{l}^{\prime} d \xi\right. \\
& \left.-\int_{0}^{1} \varphi_{i} \varphi_{j}^{\prime \prime} \int_{\xi}^{1}\left(A_{f}^{*}+\rho^{*} A_{p}^{*}\right) \int_{0}^{\xi} \varphi_{k}^{\prime} \varphi_{l}^{\prime} d \xi\right)
\end{aligned}
$$

The final matrix form of motion equations will be as follows:

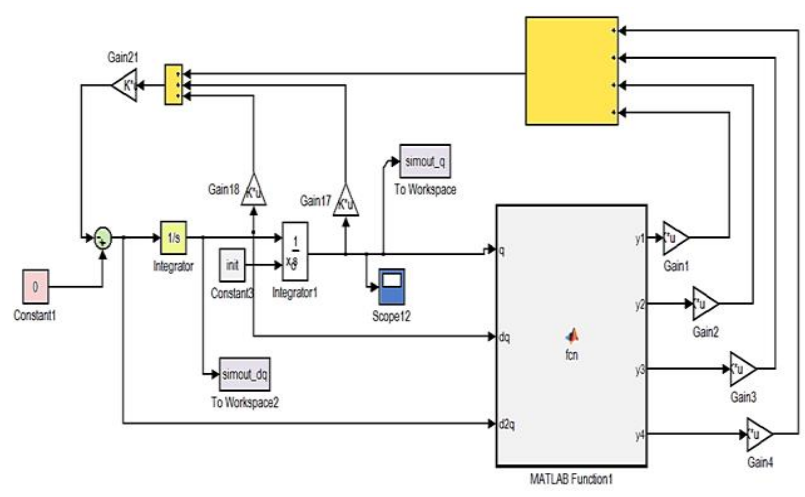

Figure 3. Simulink model of nonlinear differential equation solution

$$
\begin{aligned}
& {\left[m_{i j}\right]_{N \times N}\left\{\begin{array}{l}
\ddot{q}_{1} \\
\ddot{q}_{2} \\
\ldots \\
\ddot{q}_{N}
\end{array}\right\}_{N \times 1}+\left[c_{i j}\right]_{N \times N}\left\{\begin{array}{l}
\dot{q}_{1} \\
\dot{q}_{2} \\
\ldots \\
\dot{q}_{N}
\end{array}\right\}_{N \times 1}} \\
& +\left[k_{i j}\right]_{N \times N}\left\{\begin{array}{l}
q_{1} \\
q_{2} \\
\ldots \\
q_{N}
\end{array}\right\}_{N \times 1}+\left[B_{i j k l}\right]_{N \times N^{3}}\left\{\begin{array}{l}
q_{1} q_{1} q_{1} \\
q_{1} q_{1} q_{2} \\
\ldots \\
q_{N} q_{N} q_{N}
\end{array}\right\}_{N^{3} \times 1} \\
& +\left[D_{i j k l}\right]_{N \times N^{3}}\left\{\begin{array}{l}
q_{1} q_{1} \dot{q}_{1} \\
q_{1} q_{1} \dot{q}_{2} \\
\ldots \\
q_{N} q_{N} q_{N}
\end{array}\right\}_{N^{3} \times 1} \\
& +\left[E_{i j k l}\right]_{N \times N^{3}}\left\{\begin{array}{l}
q_{1} \dot{q}_{1} q_{1} \\
q_{1} \dot{q}_{1} q_{2} \\
\ldots \\
q_{N} \dot{q}_{N} \dot{q}_{N}
\end{array}\right\}_{N^{3} \times 1} \\
& +\left[F_{i j k l}\right]_{N \times N^{3}}\left\{\begin{array}{l}
q_{1} q_{1} \ddot{q}_{1} \\
q_{1} q_{1} \ddot{q}_{2} \\
\ldots \\
q_{N} q_{N} \ddot{q}_{N}
\end{array}\right\}_{N^{3} \times 1}\left(\begin{array}{l}
0 \\
0 \\
0 \\
0
\end{array}\right\}_{N \times 1}
\end{aligned}
$$

\section{Results and Discussion}

Numerical methods must be used to solve the equations obtained in the relation. In this study, MATLAB software and Simulink part have been used to solve these equations. The equations in Simulink are solved by Runge-Kutta method. The time step and tolerance are also considered one thousandth. The values of entry of a matrix the coefficients are calculated by the codes written in MATLAB. The figure shows the Simulink model used.

First, it is necessary to evaluate the results with the work of other researchers, and for this purpose, two diagrams of the figures are presented is shown in Figure 4. The diagram of critical velocity changes in terms of coefficient using different modes for solution.

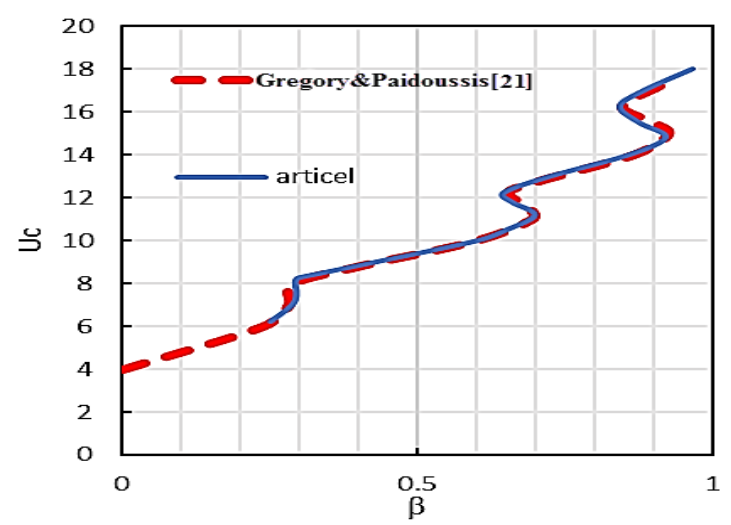

Figure 4. Evaluate results with other research work in ref [23] 
According to Figure 5, as the number of modes used in the Galerkin method for solving increases, the jumps that appear in the diagram increase, and the location of the jumps displacement to the left of the diagram. Figures 6 are shown to investigate the effect of gravity and the length of the tube on the critical velocity of the diagrams. As mentioned, to solve the nonlinear equations, the Simulink model is created according to the figure, which solves the differential equations discretized by the Galerkin method by the Rang-Kota numerical method. To ensure the correctness of the nonlinear equations, as well as the correctness of the written program for integral calculations and the Simulink model, since the results have not been worked on the cone tube so far, the results for the cylindrical tube state are compared with the research of Wang et al [24]. The comparison is shown in Figure 5. (Dimensional parameters used for evaluation: $\beta=0.213$, $\gamma=26.75)$.

It can be seen that the results are in good agreement with each other. In both graphs the critical velocity is about 8.5 .
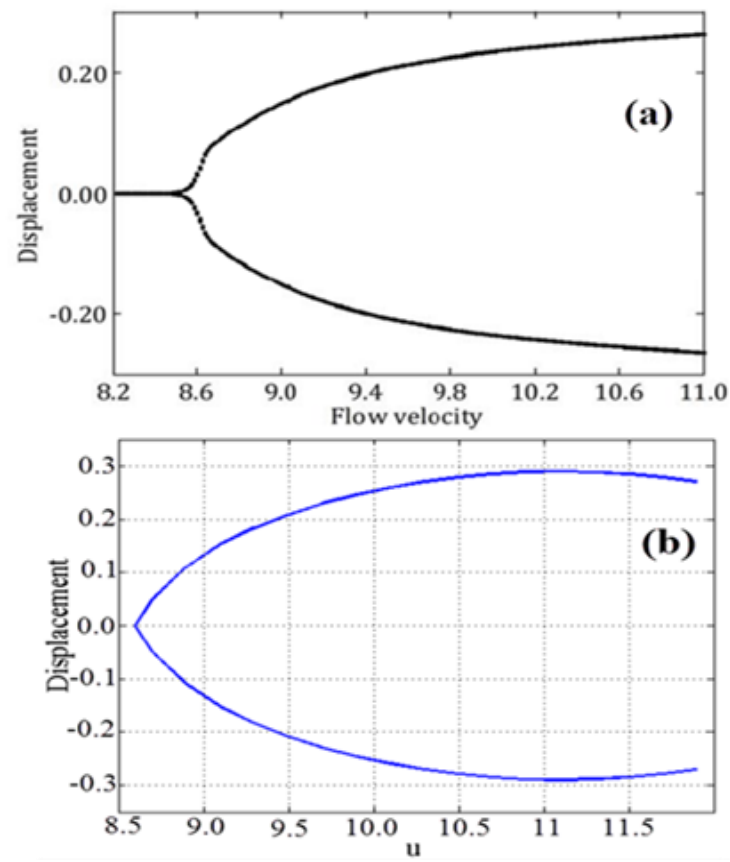

Figure 5. Diagram of the range of motion of a cylindrical tube after the occurrence of instability with a change in velocity

(a) Wang et al [24] (b) current article.

Table 5.Default values of dimensionless parameters.

\begin{tabular}{|c|c|}
\hline Parameter & Value \\
\hline $\mathrm{X}$ & 40 \\
\hline$\gamma$ & 0 \\
\hline$\rho^{*}$ & 2 \\
\hline$\lambda$ & 0.5 \\
\hline $\mathrm{I}^{*}$ & 2 \\
\hline $\mathrm{Ap}^{*}$ & 0.14 \\
\hline $\mathrm{Af}^{*}$ & 0.11 \\
\hline
\end{tabular}

The values of the $\beta_{\mathrm{T}}$ and $\alpha_{\mathrm{i}}$ parameters are also indicated in each figure. Due to the fact that with increasing $\beta$ coefficient, the modes that lead to system instability change, and considering that according to the observations of this change, the modes move towards higher modes, to see the jumps in the diagram, the modes used for solving must be increased. Otherwise jumps that occur at higher $\beta$ coefficients will not appear in the graph.

According to Figure 6, as the number of modes used in the Galerkin method to solve increases, the number of jumps that appear in the graph increases and the location of the jumps shifts to the left of the graph. To investigate the effect of the direction of gravity and the length of the tube on the critical velocity is shown in Figures 7 and 8 . $\left(\alpha_{\mathrm{e}}=0, \beta=0.5, \gamma_{(\mathrm{H})}=0.00251 \times(\mathrm{X} / 2)^{3}\right)$

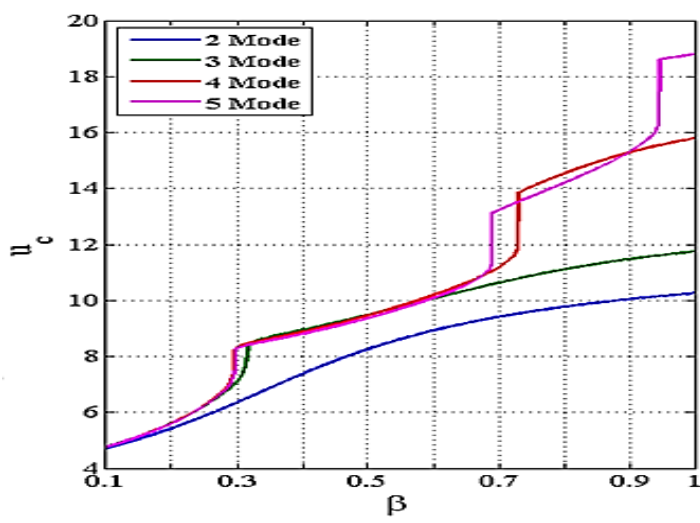

Figure 6. Critical velocity changes in different modes.

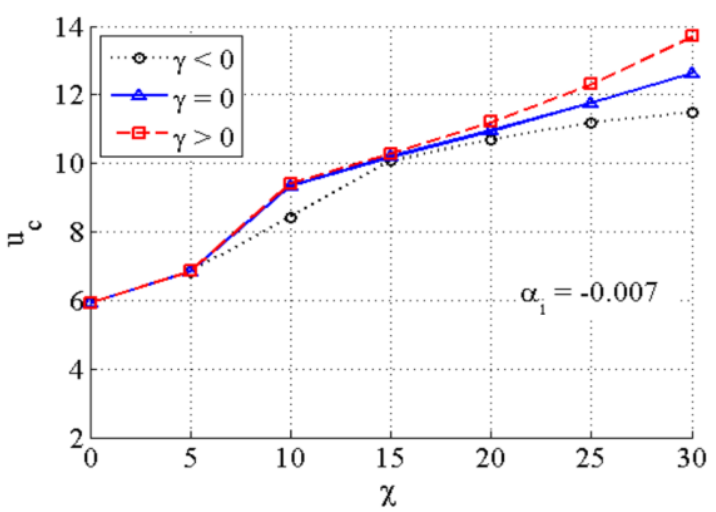

Figure 7. The Effect of gravity and the length of the tube.

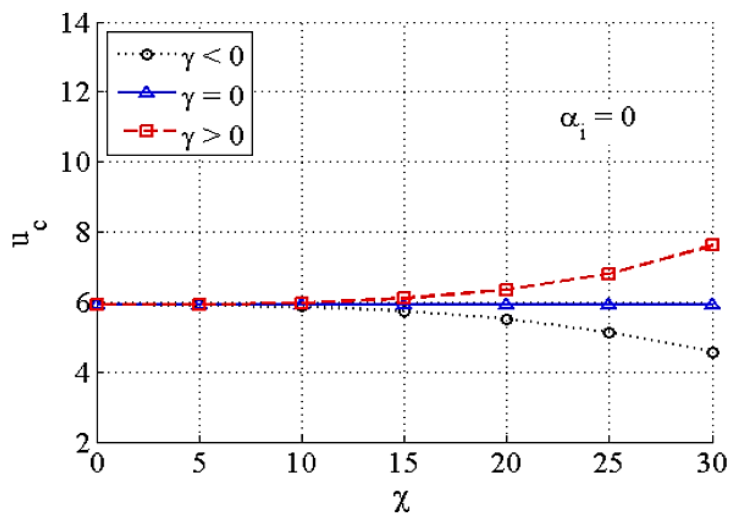

Figure 8. The Effect of gravity and the length of the tube. 
As can be seen in Figures 7 and 8, the negative $\gamma$ makes the system more unstable because the critical speed of the fluid is reduced. As $\gamma$ increases, the critical speed of the fluid increases and the system becomes more stable. This effect is the same for conical and cylindrical pipes. It should be noted that $\alpha_{i}$ and $\alpha_{e}$ indicate the slope of the inner and outer wall of the pipe. Since $\gamma$ is negative, it means that the free end of the tube is above the fixed end and the fluid flow is upwards. Therefore, the weight of the pipe and the fluid increases the tendency to deflection and instability in the pipe, thus becoming unstable at a lower speed. Fluid velocity is one of the main parameters of the problem. In the figure, the effect of velocity on changes in the amplitude of displacement after instability is shown in Figure 9. At high speeds, the predicted amplitude difference in different modes increases. In nonlinear systems, it is necessary to consider more modes to model the systems until the response is convergent. The speed limit of 11 differences is limited but then increases.

In Figure 10, the convergence is greater for the $\beta$ value, and the fourth mode is well responsive. Therefore, less modes are required for $\beta$ in modeling.

In both Figures 11 and 12, the range of the studied velocity is from the beginning of the critical velocity to twice the critical velocity. The range of motion for a conical tube with different internal angles and with different $\beta_{\mathrm{T}}$ coefficients is plotted in the following figures.

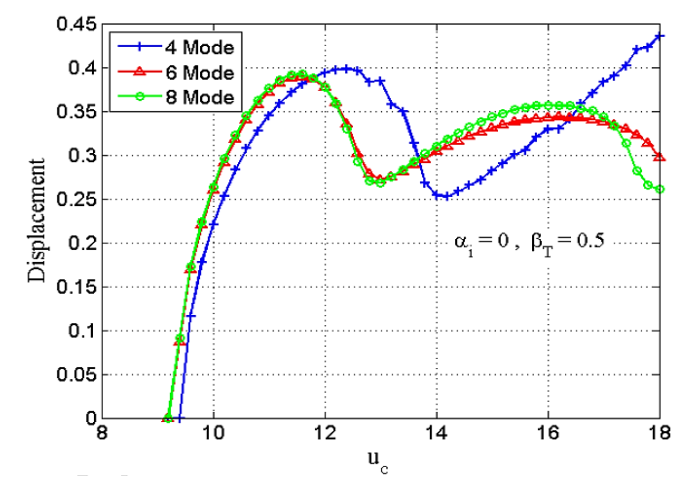

Figure 9. The Effect of velocity on changes in the amplitude of displacement $\left(a_{i}=0, \beta_{T}=0.5\right)$

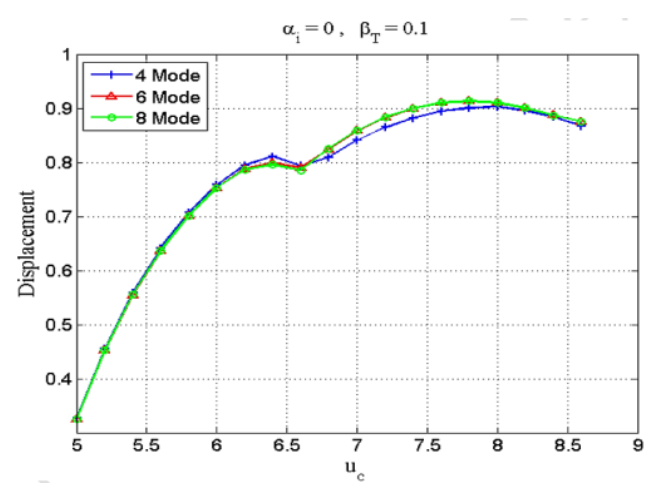

Figure 10. The Effect of velocity on changes in the amplitude of displacement $\left(a_{i}=0, \beta_{T}=0.1\right)$
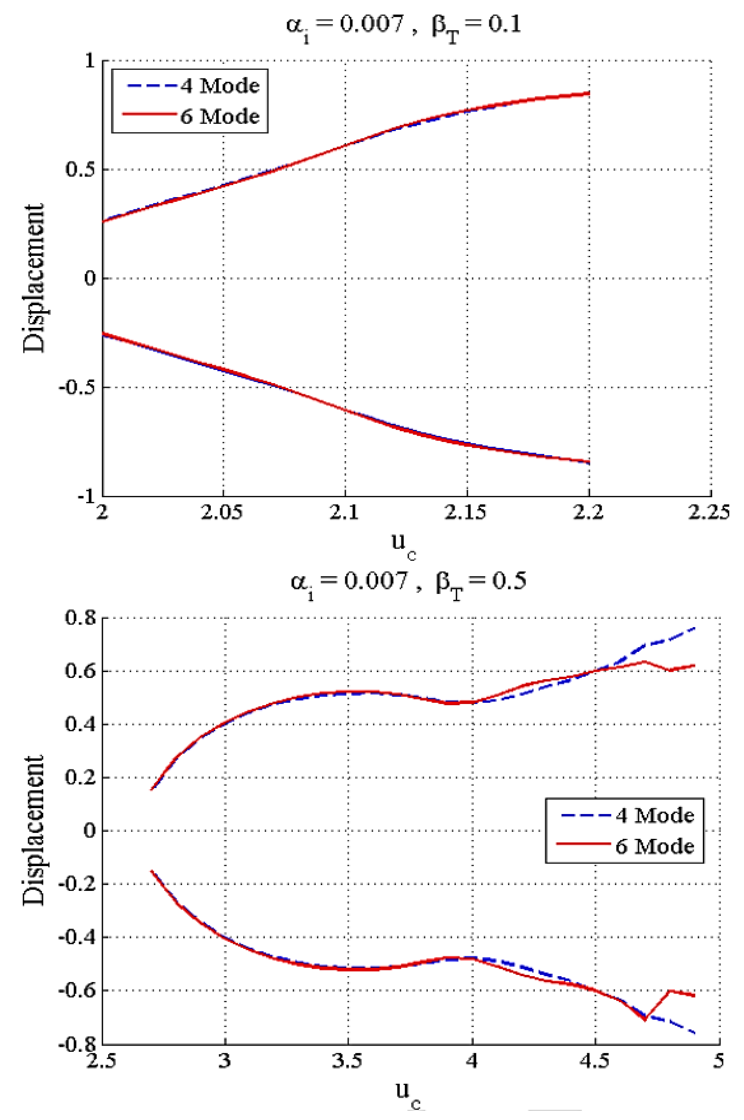

Figure 11. The Effect of velocity on changes in the amplitude of displacement $\left(a_{i}=0.007, \beta_{T}=0.1\right.$ and 0.5 )

According to Figure 12, the difference in response between the upper and lower modes in $\beta_{\mathrm{T}}$ is further increased. The effect of changing the internal slope angle of the conical, the diagram shows the maximum velocity displacement for different interior angles in Figure 13.

In Figures 14 and 15, the wider the internal angle of the wall, the lower the range of oscillations, and the narrower the internal angle, the higher the rate of growth of the oscillation range. Also, in lower beta coefficients, the scales obtained after instability have a larger size than more beta. Figure 16 shows the natural frequency of the system in terms of fluid velocity at different $L / h$ (h is the shell thickness $\left(R_{s}-r_{s}\right)$ ). As the parameter increases, the lateral ratio of the natural frequency of the system decreases and the damping frequency of the system increases. The critical velocity of the fluid is reduced and the system becomes prone to buckling.

Weight percentage of carbon nanotubes is another parameter of this study, which is its effect on the Dimensionless frequency shown in Figure 17. increasing the Weight percentage of carbon nanotubes increases the frequency of the tube. Increasing the carbon nanotubes increases the strength of the tube and thus reduces the displacement amplitude. 


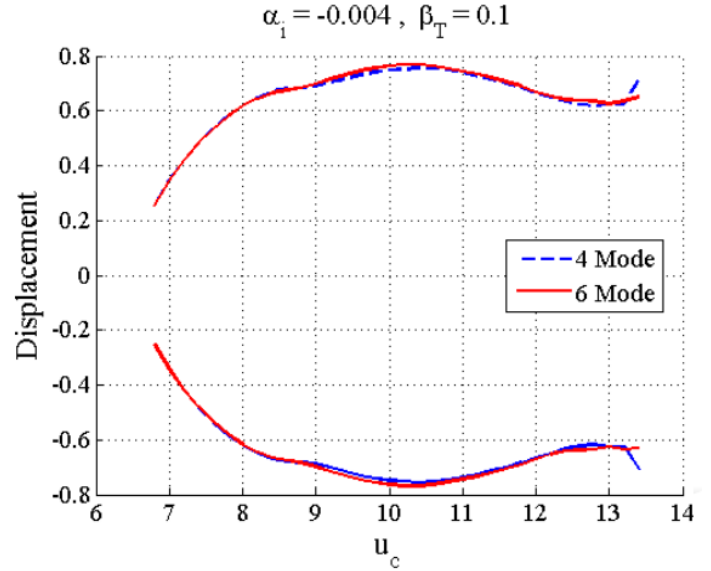

$\alpha_{\mathrm{i}}=-0.004, \beta_{\mathrm{T}}=0.5$

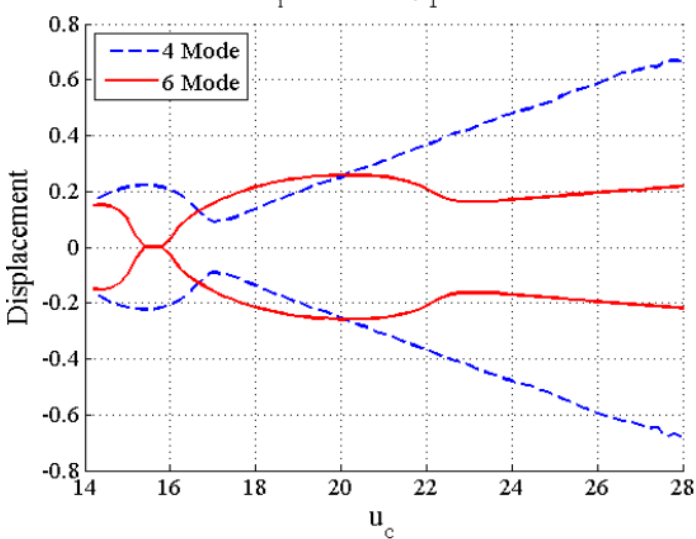

$\alpha_{\mathrm{i}}=-0.007, \beta_{\mathrm{T}}=0.1$

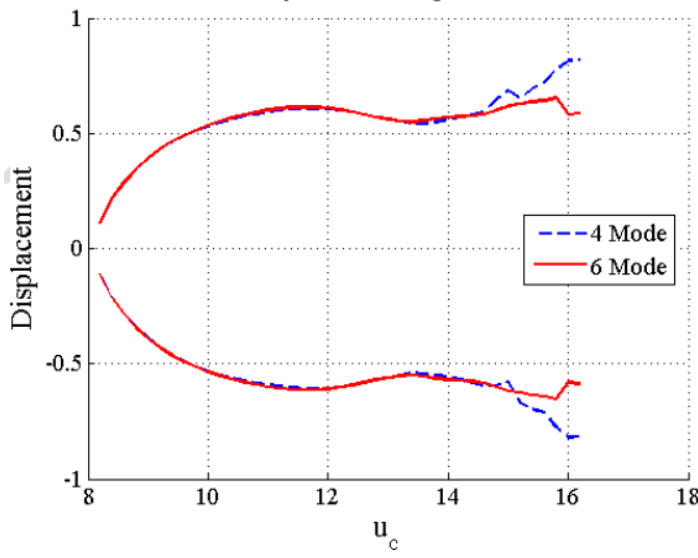

$\alpha_{\mathrm{i}}=-0.007, \beta_{\mathrm{T}}=0.5$

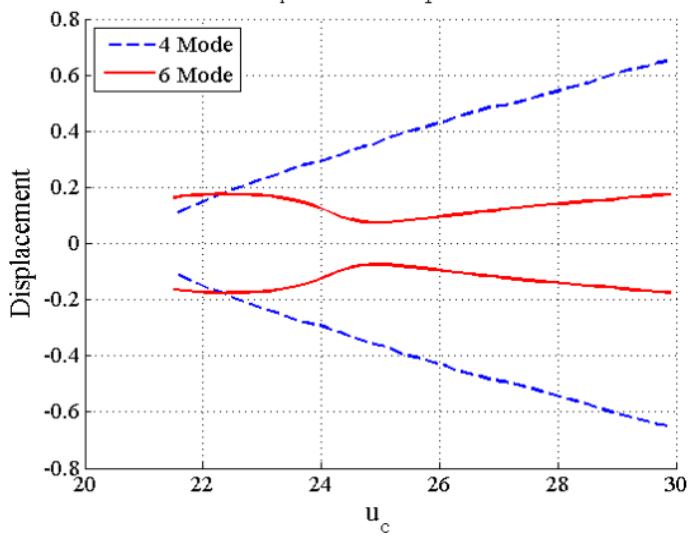

Figure 12. The difference in response between high and low modes in $\beta_{\mathrm{T}}$.
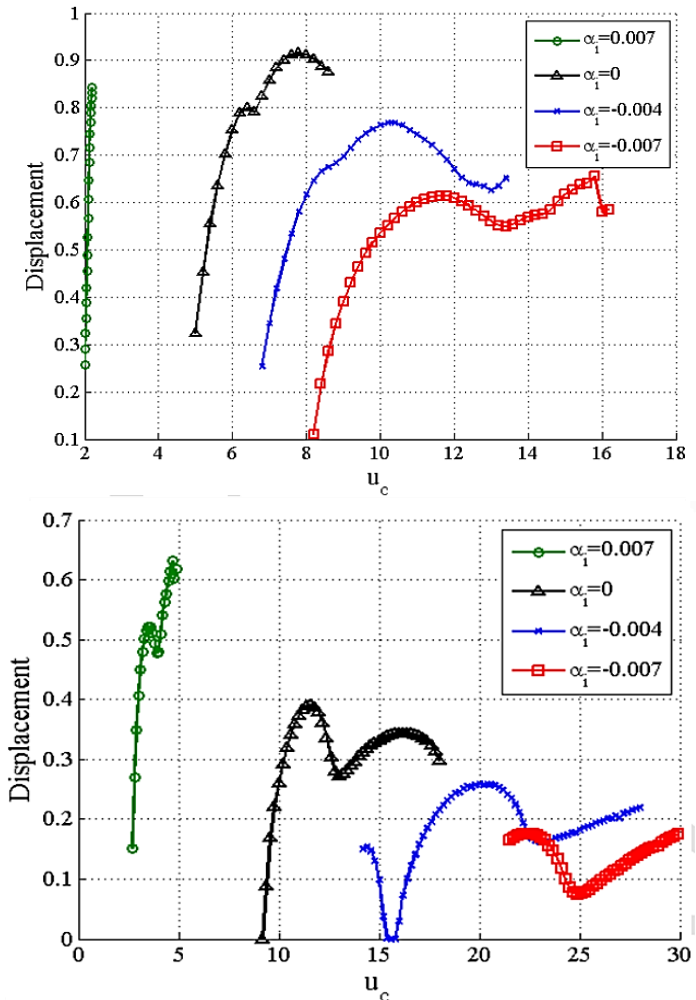

Figure 13. Maximum velocity displacement for different interior angles.

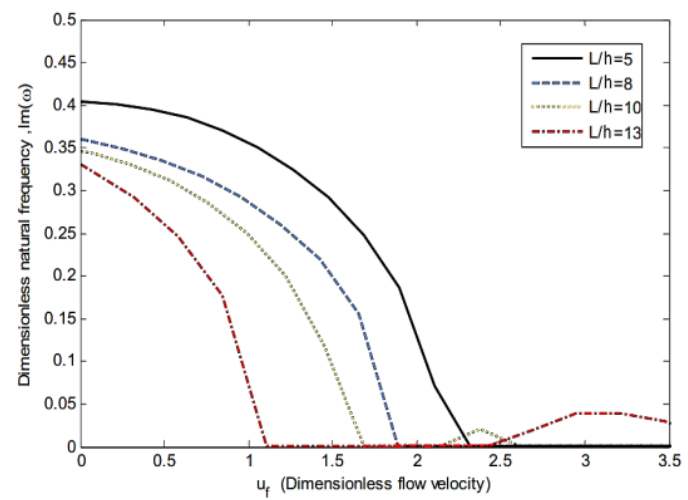

Figure 14. Dimensionless natural frequency relative to flow rate.

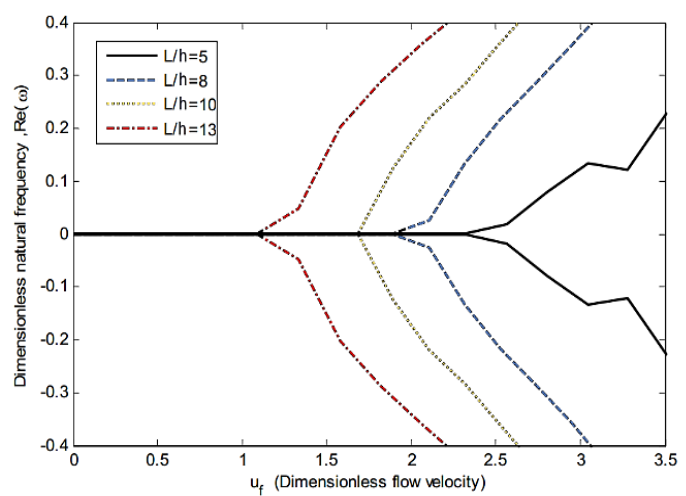

Figure 15. Dimensionless natural frequency relative to flow rate. 


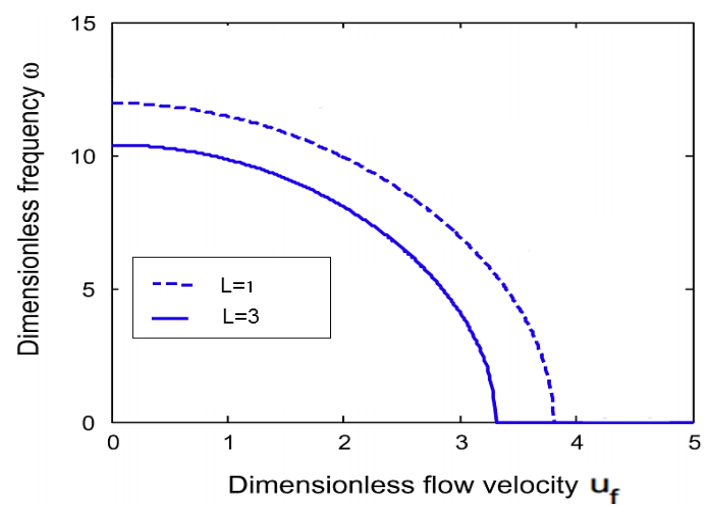

Figure 16. Natural frequency of the fluid velocity at different

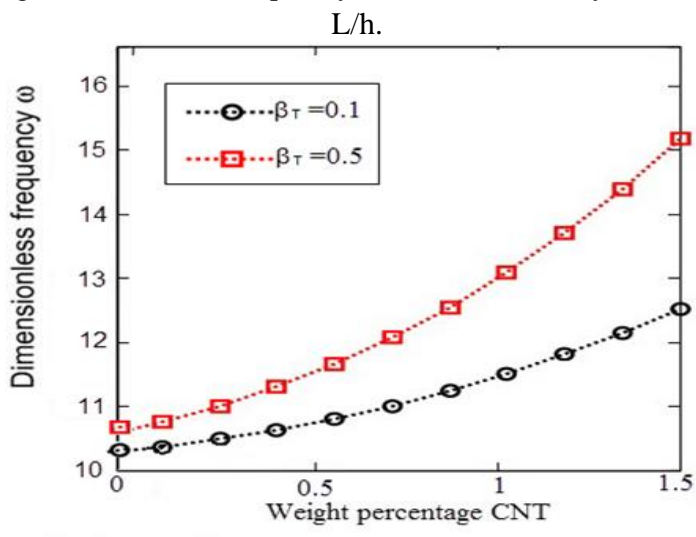

Figure 17. Frequency change by CNT weight percentage change.

\section{Conclusions}

In all sections and by changing all the parameters, it has been observed that by increasing the fluid velocity, the natural frequency of the system decreases in all vibration modes and reaches zero. At this point, the critical velocity of the fluid is defined. From this velocity onwards the system becomes unstable and prone to buckling. In addition, as the fluid velocity increases, the amplitude of the vibration increases and the nonlinear effects of the system increase, so more modes are needed to converge the responses. As the internal angle progresses toward the opening of the inner wall, the range of oscillations decreases. It also has oscillation amplitude in lower $\beta$ ranges and higher growth rates. The Runge-Kutta method is done with MatlabSimulink software and a comparison between the results of this method with the solution methods used by other researchers is shown to be a good match. In a conical tube, the $\beta T$ coefficient increases with increasing inner diameter along the tube and the system becomes more stable. Increasing the length of the pipe makes the opening conical pipe more stable and the closing conical pipe more unstable. The change in length has no effect on the stability of the cylindrical tube.

\section{Declaration}

The author(s) declared no potential conflicts of interest with respect to the research, authorship, and/or publication of this article. The author(s) also declared that this article is original, was prepared in accordance with international publication and research ethics, and ethical committee permission or any special permission is not required.

\section{References}

1. Webster, Aaron, Frank Vollmer, and Yuki Sato. Probing biomechanical properties with a centrifugal force quartz. crystal microbalance. Nature communications, 2014. 5(1): p. $1-8$.

2. Lu, Ze-Qi, Kai-Kai Zhang, Hu Ding, and Li-Qun Chen. Internal resonance and stress distribution of pipes conveying fluid in supercritical regime. International Journal of Mechanical Sciences, 2020. 186 (2020): 105900.

3. Ge, Xinbo, Yinping Li, Xilin Shi, Xiangsheng Chen, Hongling Ma, Chunhe Yang, Chang Shu, and Yuanxi Liu. Experimental device for the study of liquid-solid coupled flutter instability of salt cavern leaching tubing. Journal of Natural Gas Science and Engineering, 2019. 66: p. 168-179.

4. Amabili, M., K. Karagiozis, M. P. Païdoussis. Effect of geometric imperfections on non-linear stability of circular cylindrical shells conveying fluid. International Journal of Non-Linear Mechanics, 2009. 44(3): p. 276-289.

5. Liao-Liang, K. Wang, Y. Flow-induced vibration and instability of embedded double-walled carbon nanotubes based on a modified couple stress theory. Physica E: Lowdimensional Systems and Nanostructures, 2011. 43(5): 1031-1039.

6. Sadeghi, M. Dynamics of cantilevered pipes conveying fluid. Part 3: Three-dimensional dynamics in the presence of an end-mass. Journal of Fluids and Structures, 2007. 23(4): p. 589-603.

7. Ghayesh, Mergen H., Michael P. Païdoussis, M. Amabili. Nonlinear dynamics of cantilevered extensible pipes conveying fluid. Journal of Sound and Vibration, 2013. 332(24): 6405-6418.

8. Farajpour, A. Nonlinear mechanics of nanotubes conveying fluid. International Journal of Engineering Science, 2018. 133: p. 132-143.

9. Liang, F. Dynamical modeling and free vibration analysis of spinning pipes conveying fluid with axial deployment. Journal of Sound and Vibration, 2018. 417: p. 65-79.

10. Lu, Ze-Qi. Nonlinear vibration effects on the fatigue life of fluid-conveying pipes composed of axially functionally graded materials. Nonlinear Dynamics, 2020. 100: p. 10911104.

11. Mohammadi, N., H. Asadi, M. M. Aghdam. An efficient solver for fully coupled solution of interaction between incompressible fluid flow and nanocomposite truncated conical shells. Computer Methods in Applied Mechanics and Engineering, 2019. 351: p. 478-500.

12. Ninh, D., Nguyen D. T. Investigation for electro-thermomechanical vibration of nanocomposite cylindrical shells with an internal fluid flow. Aerospace Science and Technology, 2019. 92: p. 501-519.

13. Khudayarov, B. A., Kh M. Komilova, F. Zh Turaev. Numerical simulation of vibration of composite pipelines conveying fluids with account for lumped masses. International Journal of Pressure Vessels and Piping, 2020. 179: 104034.

14. Sedighi, H.M. Divergence and flutter instability of magneto-thermo-elastic C-BN hetero-nanotubes conveying fluid. Acta Mechanica Sinica, 2020. 36(2): p. 381-396.

15. Bahaadini, R., M. Hosseini, M. Amiri. Dynamic stability of viscoelastic nanotubes conveying pulsating magnetic 
nanoflow under magnetic field. Engineering with Computers, 2020. p. 1-13.

16. Li, Q., Liu, W., Lu, K. and Yue, Z. Nonlinear Parametric Vibration of a Fluid-Conveying Pipe Flexibly Restrained at the Ends. Acta Mechanica Solida Sinica, 2019. 33(3): p. 327-346.

17. Prince, Peter J., John R. Dormand. High order embedded Runge-Kutta formulae. Journal of Computational and Applied Mathematics,1981. 7(1): p. 67-75.

18. Rahmani, M., A. Moslemi Petrudi. Analytical Investigation of the Vibrational and Dynamic Response of Nano-Composite Cylindrical Shell Under Thermal Shock and Mild Heat Field by DQM Method. Journal of Modeling and Simulation of Materials, 2020. 3(1): p. 22-36.

19. Zhu, P., Lei, Z.X., Liew, K.M. Static and free vibration analyses of carbon nanotube-reinforced composite plates using finite element method with first order shear deformation plate theory. Composite Structures, 2012. 94(4): p. 1450-1460.

20. Morovvati, M. R., \& Mollaei-Dariani, B. The formability investigation of CNT-reinforced aluminum nano-composite sheets manufactured by accumulative roll bonding. The International Journal of Advanced Manufacturing Technology, 2018. 95(9-12): 3523-3533.

21. Benjamin, T.B. Dynamics of a system of articulated pipes conveying fluid. I. Theory. Proceedings of the Royal Society of London. Series A, 1961. 261: p. 457-486.

22. Stoker, J. J. Nonlinear elasticity. Gordon,Breach, 1968.

23. Gregory, R. W., and M. P. Paidoussis. Unstable oscillation of tubular cantilevers conveying fluid II. Experiments. Proceedings of the Royal Society of London. Series A. Mathematical and Physical Sciences,1966. 293(1435): p. 528-542.

24. Wang, L., Z. Y. Liu, A. Abdelkefi, Y. K. Wang, and H. L. Dai. Nonlinear dynamics of cantilevered pipes conveying fluid: towards a further understanding of the effect of loose constraints. International Journal of Non-Linear Mechanics, 2017. 95: p. 19-29. 